\title{
RANDOM VIBRATION ANALYSIS ON INSTALLATION OF AN ENVIRONMENTAL CONTROL SYSTEM COMPONENT IN FIGHTER AIRCRAFT
}

\author{
Kishen Karumbaiah B J ${ }^{1}$, Y J Suresh ${ }^{2}$, Basava $\mathbf{T}^{3}$ \\ ${ }^{1}$ Assistant Professor in Mechanical Engineering, Coorg Institute of Technology, Ponnampet, Karnataka State, India \\ ${ }^{2}$ Professor in Mechanical Engineering, Jawaharlal Nehru National College of Engineering, Shimoga, Karnataka \\ State, India \\ ${ }^{3}$ Professor in Mechanical Engineering, Sri Dharamastala Manjunatheswara Institute of Technology, Ujire, Karnataka \\ State, India
}

\begin{abstract}
Random vibration analysis is a major requirement to predict safe flight envelopes and to decide random vibration testing conditions of newly designed or modified aircraft structures. In order to achieve reliable random analysis technique it is necessary to obtain a good correlation between numerical (finite element method) and analytical techniques. This paper presents the determination of random stress in aircraft structure by frequency domain approach. Validation of the approach is shown by performing random analysis on a single degree of freedom system and comparing the results by both analytical approach and finite element technique. On determination of random stresses the importance of random analysis in aircraft structure is mentioned as it leads to fatigue failure of the components.
\end{abstract}

Keywords: Random Vibration, Power Spectral Density, Root Mean Square Value, Random Stress, Frequency $* * *$

\section{INTRODUCTION}

The work presented in this paper aims at determining the random stress values in heat exchanger assembly attachment members in a fighter aircraft by super imposing white noise spectral density value on frequency response transfer function for the given structure excited by unit harmonic base excitation. The variation of energy content in the structure is found out by determining the area under the spectral density During operation of a Military Fighter Aircraft considerable amount of vibration is experienced due to noise of engines, aerodynamic turbulent flow around the wings and fuselage leading to non- stationary pressure fluctuations on the aircraft structure which gets transferred to the primary load carrying structures of the aircraft called bulkheads, and further to the attaching members. As the flight loads are non periodic and do not have a pre-specified nature of occurrence they are difficult to be characterized and defined. The only way to specify such kind of erratic behaviour is statistically, and is termed as random mechanical vibrations. Continuous exposure to such oscillating loads results in increase of stress levels which are above the designed static and dynamic stress limits leading to failure of the member due to fatigue. This has necessitated the need for random vibration analysis on aircraft structures and the determination of random stresses.

Random vibration analysis can be classified into two main categories, time domain and frequency domain approach. Time domain approach aims at determining the statistical properties (namely probability distribution, correlation, and cross-correlation) based on ensemble random vibration data. On the other hand, frequency domain approach offers the knowledge of energy content in the signal in terms of power spectral density and root mean square values.

Determination of random stress and fatigue life characteristics for a cantilever beam performed using finite element technique presented by Santhosh M Kumar [1] involves harmonic excitation of the beam and superimposition of white noise to simulate random vibration environment utilizing the analysis package. Tom Irvine [2] suggested the utilization of Gaussian distribution in applying static random loads and determination of root mean square values in the time domain. Application of frequency domain approach and use of miles equation in determining root mean values is demonstrated by Daniel J. Segalman, et al. [3]. Antony J. Davenport, et al. [4] presented the advancements in random vibration simulation using $\mathrm{FE}$ package MSC PATRAN/NASTRAN. J. W Roberts, et al. [5] presents theoretical basis of an analogue method for determining random response of a structure subjected to harmonic excitations. The response of a structure to random vibration is studied by exciting the structure harmonically and superimposing the exciting signal with white noise. The variation of energy content curve in terms of root mean square (rms) value. The maximum random stress value is calculated based on the rms values. Two cases are considered, random vibration analysis on primary and secondary heat exchanger attachment members of a military fighter aircraft using MSC PATRAN/NASTRAN analysis package and random stress values at different attachment 
locations are determined. In case two random analysis is performed on a SDOF system using both FE technique and analytical relations. Determined stress values are compared to validate the finite element analysis procedure followed in both the cases.

\section{THEORY}

\subsection{Fourier Transform}

The Fourier transform of a non-periodic $l(t)$ signal, having a finite total energy, is given by the relationship,

$$
\mathrm{L}(\omega)=\int_{-\infty}^{\infty} l(t) e^{-i \omega t} \mathrm{dt}
$$

The above expression is complex, in order to represent it is therefore necessary to graphically plot; either the real and imaginary part versus the angular frequency $\omega$, or the amplitude and phase, versus the angular frequency $\omega$. The curve of amplitude versus phase is called the Fourier spectrum. As the random signals are not of finite energy, thus we can calculate only the Fourier transform of a sample of signal of duration $\mathrm{T}$ by supposing this sample is representative of the whole phenomenon. It is in addition possible, starting from the expression of $\mathrm{L}(\omega)$, to return to the temporal signal by calculation of the inverse transform.

$$
l(\mathrm{t})=\frac{1}{2 \pi} \int_{-\infty}^{\infty} l(\omega) e^{i \omega t} d \omega
$$

We can limit to the comparison of the amplitudes, neglecting phase. It is preferable to proceed with an average of the modules of Fourier transforms calculated for several signal samples (more exactly, an average of the squares of the amplitudes). This is the idea behind power spectral density.

\subsection{Power Spectral Density}

The usual way to describe the severity of damage for random vibration is in terms of its power spectral density (PSD), a measure of a vibration signal's power intensity in the frequency domain. The way to evaluate is to determine the average value of all the amplitudes within a given frequency range. Although acceleration amplitude at a given frequency constantly changes, its average value tends to remain relatively constant. Power spectral density tells us how the power of random signal is distributed over a certain bandwidth (frequency range). The PSD is usually expressed in units of acceleration as $\mathrm{g}^{2} / \mathrm{Hz}$. Random vibration analysis is usually performed over a wide range of frequency usually 20 - $2000 \mathrm{~Hz}$. Such a study does not look at a specific frequency or amplitude at a specific moment in time but rather statistically looks at a structure's response to a given random vibration environment. The square root of the area under the PSD curve gives the acceleration root mean square value (Grms), which is a qualitative measure of intensity of vibration.

\subsection{Analytical Procedure to Determine Random}

\section{Stress Value in a SDOF System [6], [7]}

A Single Degree Of Freedom (SDOF) system with a discrete mass ' $m$ ', damper element ' $c$ ' and spring element ' $k$ ' is placed on a moving base with an acceleration $\ddot{u}(t)$. The resulting displacement of the mass is $x(t)$. A relative motion $z(t)$ will be introduced which is the displacement of the mass with respect to the base.

The relative displacement is

$$
z(t)=x(t)-u(\mathrm{t})
$$

The equation of motion of the SDOF system, illustrated in the figure is

$$
m \ddot{x}(t)+c\{\dot{x}(t)-\dot{u}(t)\}+k\{x(t)-u(\mathrm{t})\}=0 .
$$

Substituting $\mathrm{Eq}$ (3) in $\mathrm{Eq}$ (4) the equation of motion for the SDOF system can be written as

$$
\ddot{z}(t)+2 \zeta \omega_{n} \dot{z}(t)+\omega_{n}^{2} z(t)=-\ddot{u}(t(5)
$$

Where $\zeta$ and $\omega_{n}$ represents the damping ratio and angular natural frequency respectively, $\ddot{z}(t)$ and $\dot{z}(t)$ represents relative acceleration and relative velocity the mass with respect to base acceleration excitation respectively.

Solving in frequency domain means forced response characteristics (particular integral) of the SDOF system are taken into account, no initial conditions are taken into account, they are assumed to be damped out. This type of response is called forced response, frequency response or steady state response.

By using the Fourier Transform Technique

The Fourier transform of the second derivative of $z(\mathrm{t})$, the acceleration $\ddot{z}(t)$ is

$$
\mathrm{F}\{\ddot{Z}(t)\}=(j \omega)^{2} \mathrm{Z}(\omega)=-\omega^{2} \mathrm{Z}(\omega)=\int_{-\infty}^{\infty} \ddot{Z}(t) e^{-j \omega t} d t
$$

The Fourier transform of Eq (5) will lead to the following equation of motion in frequency domain

$$
\left[-\omega^{2}+2 j \zeta \omega \omega_{n}+\omega_{n}^{2}\right] \mathrm{Z}(\omega)=-\ddot{U}(\omega
$$

Or

$$
Z(\omega)=\frac{-\ddot{U}(\omega)}{-\omega^{2}+2 j \zeta \omega \omega_{n}+\omega_{n}^{2}}=-H(\omega) \ddot{U}(\omega) .
$$

From Equation (8) we can write the frequency response function (FRF) $H(\omega)$ as,

$$
H(\omega)=\frac{1}{\sqrt{\left[1-\left(\frac{\omega}{\omega_{n}}\right)^{2}\right]^{2}+\left[2 \zeta\left(\frac{\omega}{\omega_{n}}\right)\right]^{2}}}
$$


And

$$
|H(\omega)|^{2}=\frac{1}{\left[\left(1-\frac{\omega^{2}}{\omega_{n}^{2}}\right)^{\wedge} 2+\left(2 \zeta \frac{\omega}{\omega_{n}}\right)^{\wedge} 2\right]}
$$

The PSD function of $\ddot{u}(t)$ is given by the following equation

$$
S_{u \dddot{u}}(\omega)=\lim _{T \rightarrow \infty} \frac{1}{T} \ddot{U}(\omega) \ddot{U}^{*}(\omega) .
$$

Where $\ddot{U}(\omega)$ and $\ddot{U}^{*}(\omega)$ are complex conjugates.If the enforced Spectral Density Function is constant over the band width (i.e) $S_{u \ddot{u}}(\omega)=\mathrm{S}_{\mathrm{o}}$, then the PSD function $S_{z z}(\omega)$ of the relative motion $\mathrm{z}(\mathrm{t})$ is

$$
S_{\mathrm{zz}}(\omega)=|H(\omega)|^{2} S_{u \ddot{u}}(\omega)
$$

To find the Root Mean Square $\left(\mathrm{G}_{\mathrm{rms}}\right)$ Value we need to find the area below the PSD response curve.

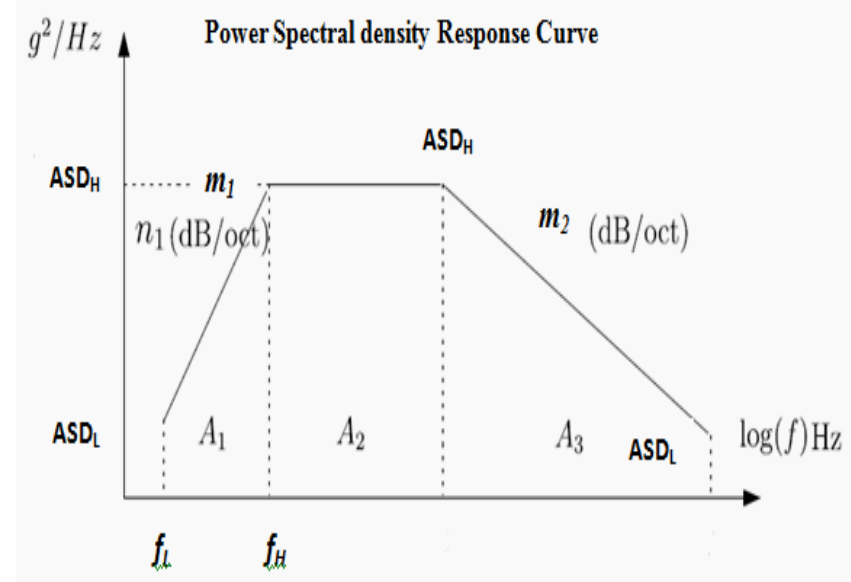

Fig 1: Example of PSD Response showing different slopes

If the Spectrum is with Positive slope area can be found using

$$
\mathrm{A}_{1}=\left\{\frac{A S D_{H} f_{H}}{m_{1}+1}\left[1-\left(\frac{f_{L}}{f_{H}}\right)^{m_{1}+1}\right]\right\}
$$

Where $\mathrm{ASD}_{\mathrm{H}}=\mathrm{PSD}$ value at Higher Frequency, $f_{H}$ $f_{L}=$ Lower Frequency

$m_{1}=$ positive slope, given as

$$
m_{1}=\frac{\log \left[\frac{A S D_{H}}{A S D_{L}}\right]}{\log \left[\frac{f_{H}}{f_{L}}\right]}
$$

If the spectrum is flat i.e with zero slope, the area is found using the expression

$$
\mathrm{A}_{2}=\operatorname{ASD}_{\mathrm{H}}\left[f_{H}-f_{L}\right]
$$

If the spectrum is with negative slope, the area is found using

$$
\mathrm{A}_{3}=\left\{\frac{A S D_{H} f_{H}}{m_{2}+1}\left[\left(\frac{f_{L}}{f_{H}}\right)^{m_{2}+1}-1\right]\right\}
$$

Where $m_{2}=$ negative slope, found out as

$$
m_{2}=\frac{\log \left[\frac{A S D_{L}}{A S D_{H}}\right]}{\log \left[\frac{F_{H}}{F_{L}}\right]}
$$

Total Area $\mathrm{A}_{\mathrm{T}}=\left(\mathrm{A}_{1}+\mathrm{A}_{2}+\right.$ .$\left.A_{n}\right)$

$$
\mathrm{G}_{\mathrm{rms}}=\sqrt{A_{T}} \quad \mathrm{~m} / \mathrm{sec}^{2}
$$

The bending stress due to random vibration can be found using Equation

$$
\sigma_{\text {Rbend }}=\frac{M_{\text {Rbend }} \times\left(\frac{t}{2}\right)}{I} . . \mathrm{N} / \mathrm{m}^{2}
$$

Bending moment $\mathrm{M}_{\text {Rbend }}=\mathrm{m} \mathrm{G}_{\mathrm{rms}} \mathrm{L}$

Where $\mathrm{m}, \mathrm{L}$ and $\mathrm{t}$ are the mass, length and thickness of the beam respectively, $G_{r m s}$ is the acceleration root mean square value.

\section{CASE STUDIES}

In this section, two case studies are presented. In the first case study, the random stresses in the primary and secondary heat exchanger assembly are found out using finite element analysis package. In the second case the validation of the applied procedure is shown by performing random analysis on a SDOF system analytically and numerically.

\subsection{Case Study I}

The primary and secondary heat exchangers assembly attachment structures of a military fighter aircraft are subjected to random vibration analysis with spectral density value specified accordance to military standard MIL-STD810D[9]. The finite element technique is applied with MSC PATRAN/NASTRAN analysis package [8]. The assembled CADD model of the heat exchanger is shown in Fig. 2. As the attaching members having contact with the bulkheads are responsible to take up the random loads in the analysis only the attachment components are considered shown in Fig.3. The finite element model of the components is prepared using linear two dimensional quad elements and the constrain points are shown in Fig. 4. Boundary conditions are provided by constraining all degrees of freedom for riveted joints and only translations are fixed at bolted joints. To simulate the random environment unit acceleration base excitation is applied in all the three mutually perpendicular directions at all the constraint points shown in Fig. 5. The application of random load is in terms of spectral density value according to MIL-STD [9] (military standard) is as shown in Fig. 6 for a band width of $20-2000 \mathrm{~Hz}$. The material used is steel with Young's modulus $210 \mathrm{GPa}$, Poissons ratio 0.3 , density $7800 \mathrm{~kg} / \mathrm{m}^{3}$ and a damping factor of 0.02 is considered. 


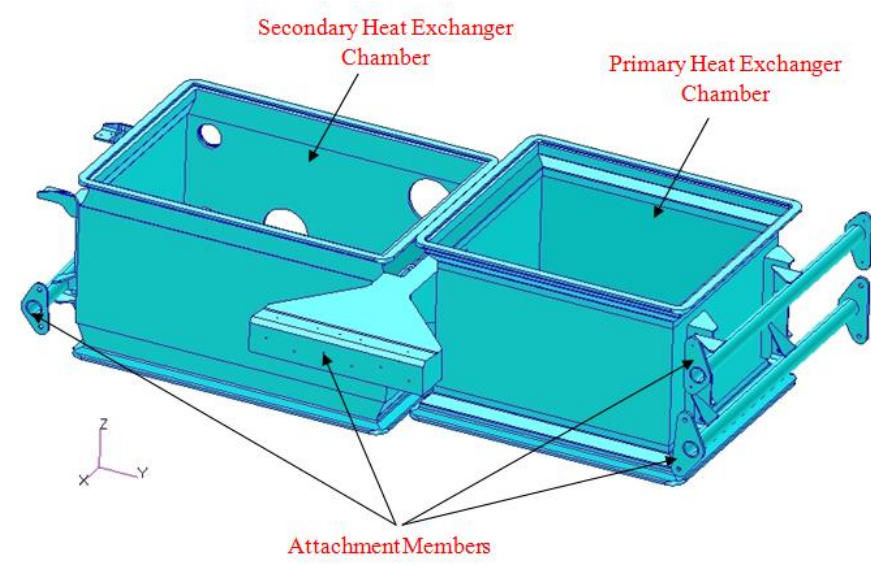

Fig 2: Assembled CADD model of Heat Exchangers

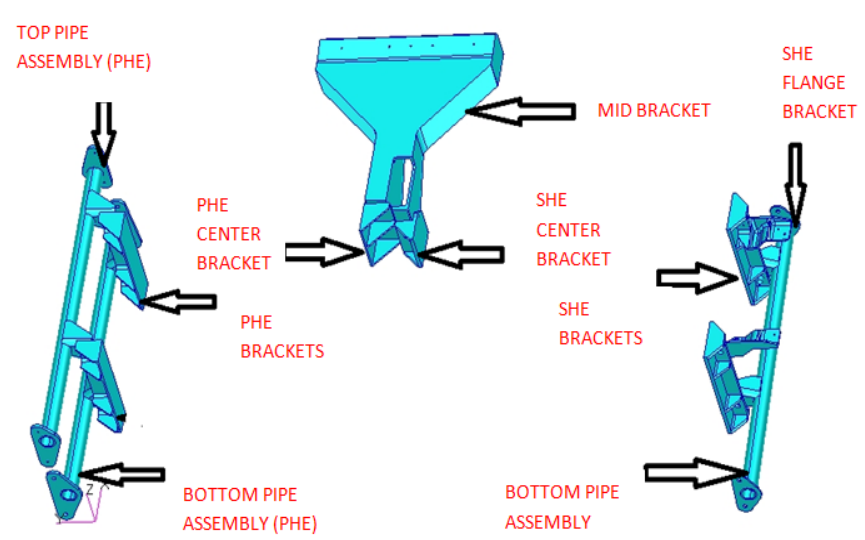

Fig 3: Attachment components considered for analysis

\section{RIVETED JOINTS}

BOLTED JOINTS

WELDED JOINTS

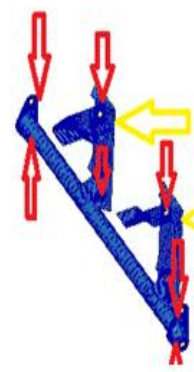

Fig 4: FE Model of attachment components
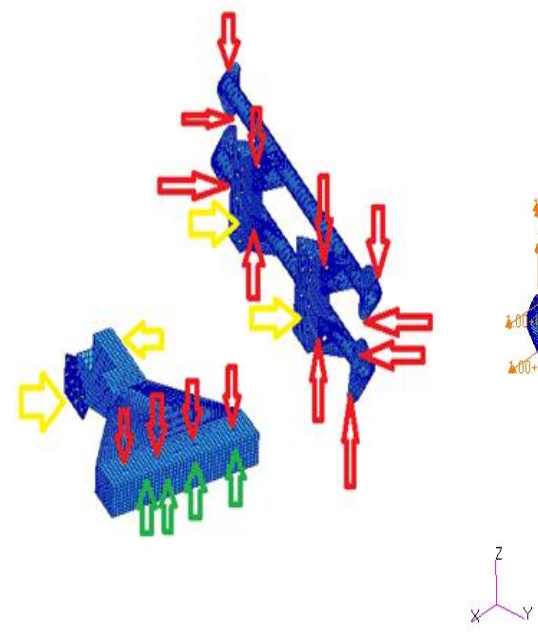
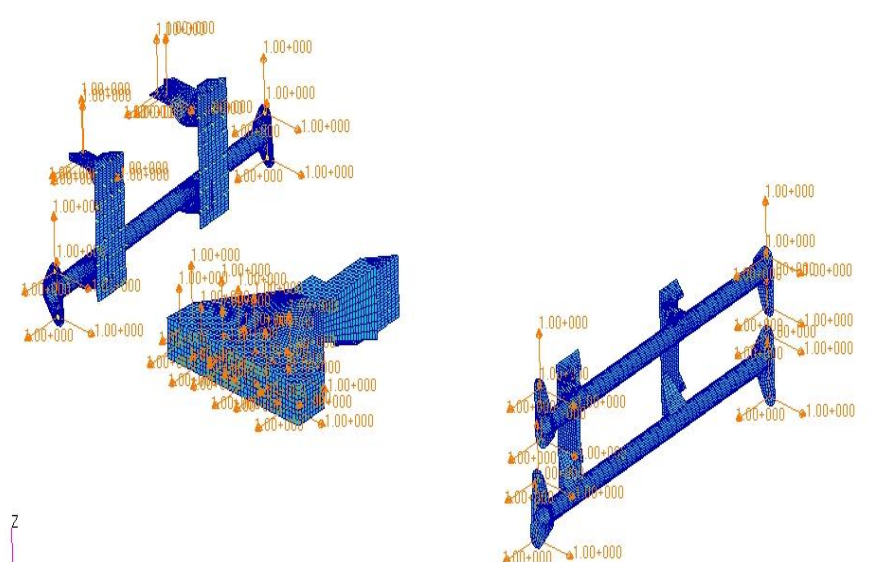

Fig 5: Application of Base Excitation at constrained points

On analysing all the attachment components it is found that the maximum random stress magnitude is approximately around 23 MPa due to $\mathrm{x}$ direction excitation. The component with the maximum stress value is shown in Fig. 7.

MSC. Patran $2010 \quad$ 17-Feb-12 13:37:43

Fringe: RMS, 16-point-stress- $x$. Stress Tensor, von Mises, At Z1

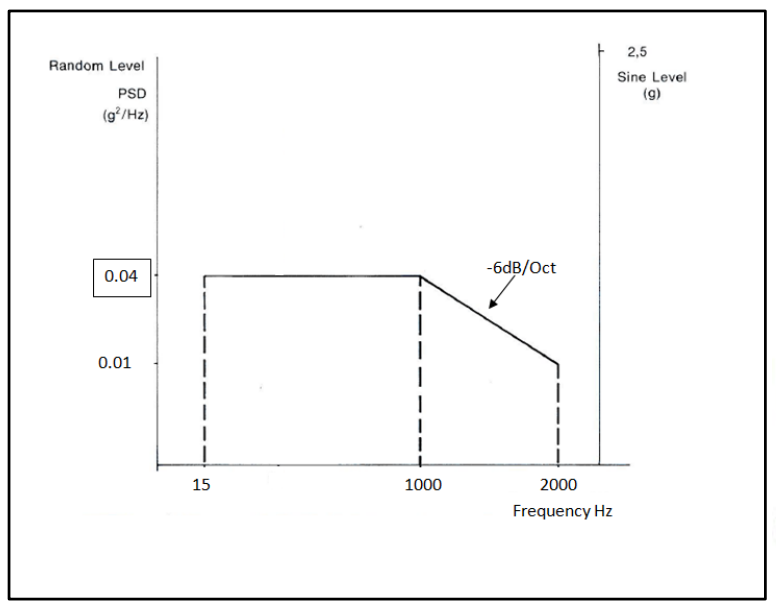

Fig 6: Random input spectrum (MIL-STD-810D)

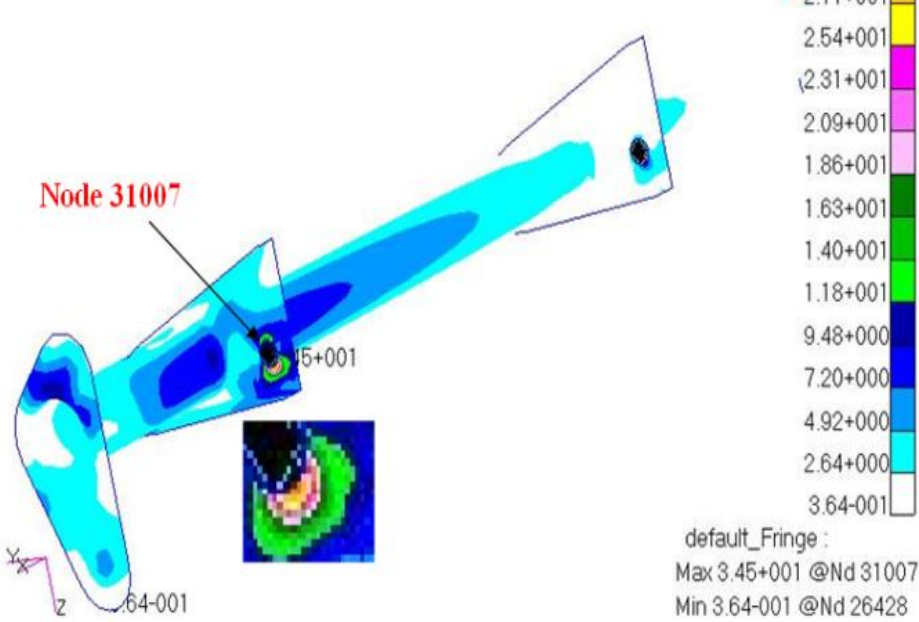

Fig 7: Random Stress fringe Plot in the attachment component 


\subsection{Case Study II}

On applying the analytical procedure to a cantilever beam with I cross-section having length equals 1 meter made up of aluminium subjected to base excitation of unit acceleration and a constant white noise spectral density value of $0.01 \mathrm{~g}^{2} / \mathrm{Hz}$ over the band width $1-101 \mathrm{~Hz}$. With a mass of 40 $\mathrm{Kg}$ is dumped at the free end and taking damping factor 0.05
On performing frequency response analysis and comparing the response plots obtained by both analytical procedures Fig. 8. and finite element technique Fig. 9. it can be observed that the peak acceleration value occurs at a frequency of $24 \mathrm{~Hz}$ which is the first natural frequency of the beam.

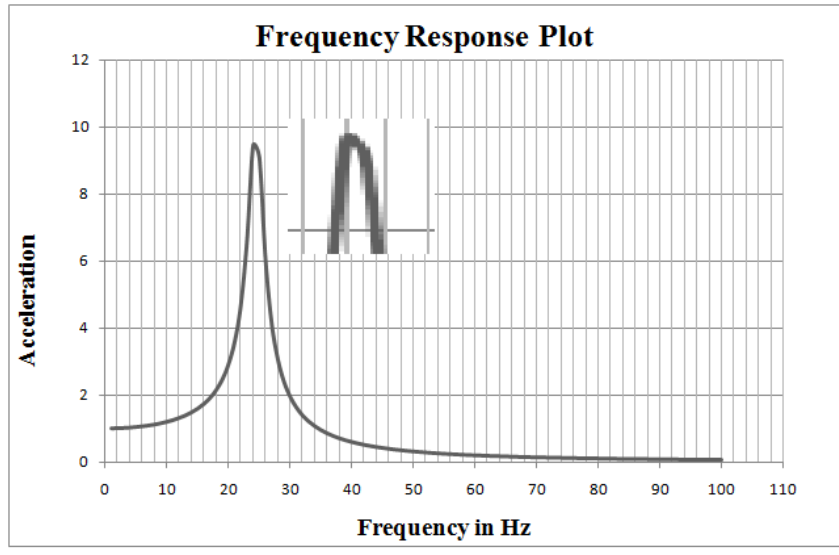

Fig 8: FRF plot obtained based on analytical values.

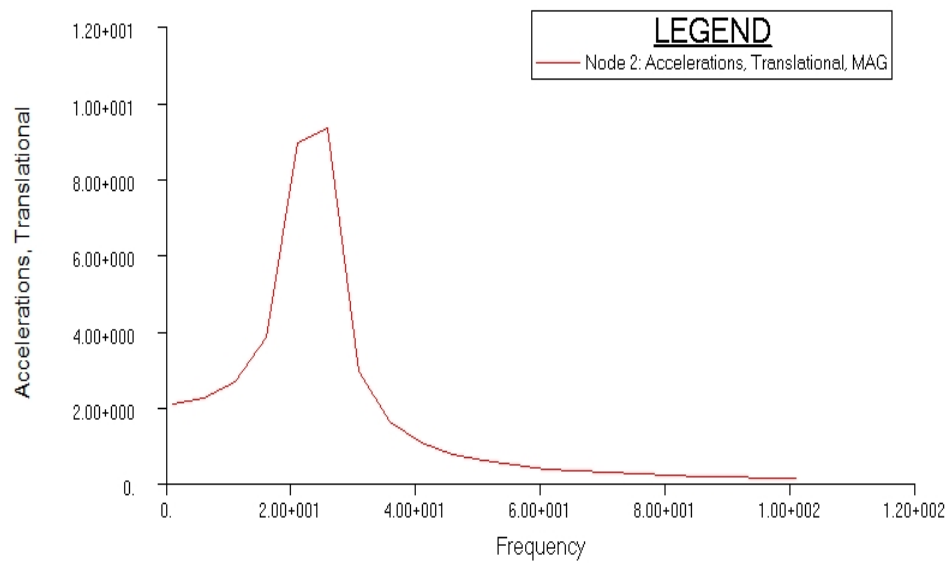

Fig 9: FRF plot obtained by FE package
Superimposing the constant white noise spectral density value on the frequency response transfer function, the analytical values of power spectral density is obtained and plotted with PSD values versus the bandwidth frequency using logarithmic scale as in Fig. 10. Similar approach using the FE package provides the spectral density response plot as shown in Fig. 11. The plots obtained from both

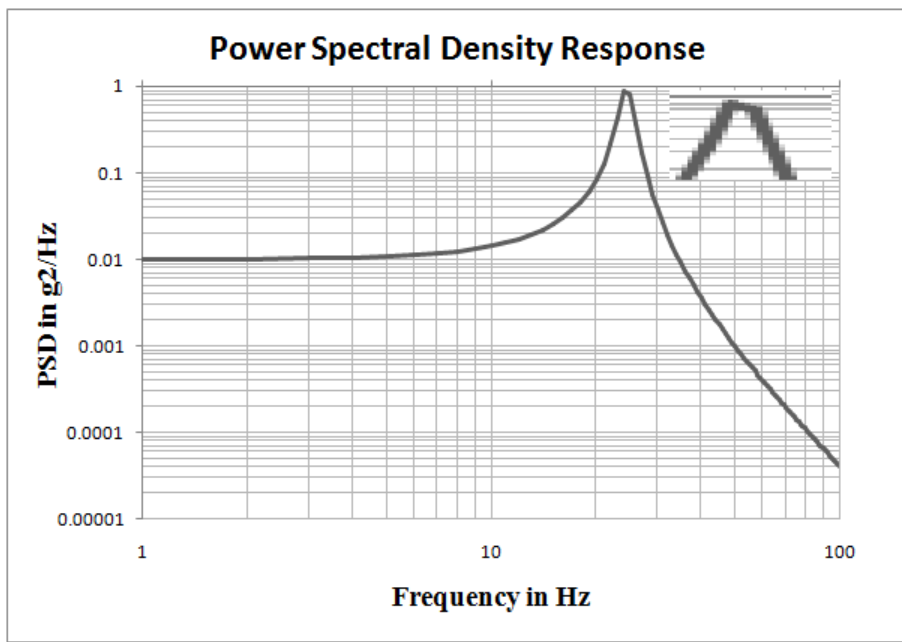

Fig 10: PSD response plot based on analytical values.

\section{CONCLUSION}

Determination of random stress values in the attachment components of heat exchangers in a fighter aircraft is performed using finite element technique by super imposing white noise spectral density on frequency response transfer function. Maximum random stress magnitude of $23 \mathrm{Mpa}$ is approaches shows the feasibility of the procedure adopted in obtaining the acceleration root mean square values in structures subjected to random vibrations. The area under the spectral density curve is calculated according the procedure explained in section 2.3. The rms value from the analytical procedure obtained is $2.095 \mathrm{~m} / \mathrm{sec}^{2}$ and that obtained by the FE package is $2.186 \mathrm{~m} / \mathrm{sec}^{2}$.

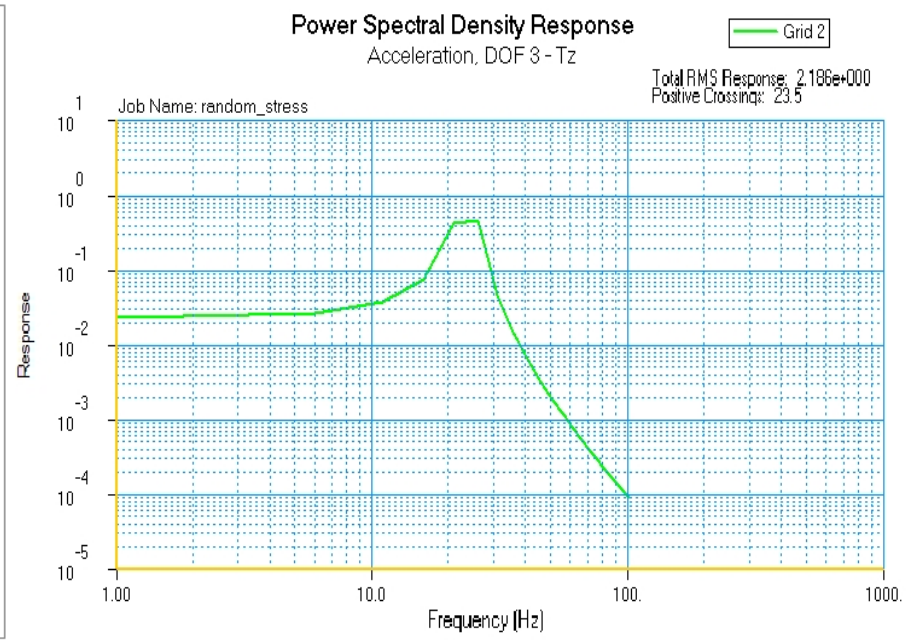

Fig 11: PSD response plot obtained by FE package.

noted down in the complete assembly structure which provides a clear knowledge of oscillating stress coming into picture during aircraft operation which gets added up to the design value of static and dynamic stresses in the aircraft structures leading to fatigue failure of the component. Hence the importance of random vibration analysis is felt in the 
dynamic design of the aircraft structure. In the second case the validation of the applied finite element procedure for the heat exchangers is shown by subjecting a SDOF system to both analytical and finite element random analysis procedures and satisfactory results are obtained.

\section{ACKNOWLEDGEMENT}

Author wishes to thank the authorities of ARDC Division, HAL (Hindustan Aeronautical Limited) Bangalore, India for granting permission and support to carry out the dissertation work.

\section{REFERENCES}

[1]. Santhosh M Kumar, "Analysing random vibration fatigue" paper presented in ANSYS Advantage. Volume II, Issue 3, 2008

[2]. Tom Irvine, "Equivalent static loads for Random Vibration" research work published in the internet by October 2010.

[3]. Daniel J. Segalman, et al, " An efficient method for calculating RMS Von Mises stress in a random vibration environment" Journal of sound and vibration vol. 230, pp 393-410, year 2000.

[4]. Antony J. Davenport, et al, "An Integrated Approach to Random Analysis Using MSC/PATRAN with MSC/NASTRAN" Notthrop Grumman Corporation, electronic sensors and systems section.

[5]. J. W. Roberts et, al, "The Simulation of Random Vibration Response by Discrete Frequency Testing" Journal of Sound and Vibration, Vol 42(4) 1975.

[6]. Jaap Wijker "Random Vibrations in Spacecraft structures Design, Theory and Applications" published by Springer publications, 2009.

[7]. Jaap Wijker "Mechanical Vibrations in Spacecraft Design" published by Springer Publications.

[8]. Release guide MSC/PATRAN and MSC/NASTRAN 2005r.

[9]. FEMCI the Book, NASA GSFE Code 542. July 2001. 